\title{
Important consequences of atomic diffusion inside main- sequence stars: opacities, extra-mixing, oscillations
}

\author{
M. Deal ${ }^{1,2,3}$, S. Vauclair ${ }^{2,3}$, and O. Richard ${ }^{1}$ \\ ${ }^{1}$ LUPM, Université de Montpellier, France \\ ${ }^{2}$ Université de Toulouse, UPS-OMP, IRAP, Toulouse, France \\ ${ }^{3}$ CNRS, IRAP, 14 avenue Edouard Belin, F-31400 Toulouse, France
}

\begin{abstract}
Atomic diffusion, including the effects of radiative accelerations on individual elements, leads to important variations of the chemical composition inside stars. The accumulation of important elements in specific layers leads to a local increase of the average opacity and to hydrodynamic instabilities that modify the internal stellar structure. This can also have important consequences for asteroseismology.
\end{abstract}

\section{Introduction}

Atomic diffusion is a fundamental process which occurs in all stars, except when prevented by large mixing processes. Due to selective effects, each ion undergoes individual motions during small timescales, before sharing its momentum through collisions. These short motions, statistically added, lead to atomic diffusion processes, which may have important consequences for the global stellar structure. Most stellar evolution codes include helium and sometimes heavy elements gravitational settling. Very few include the computations of the selective radiative accelerations of individual species in a complete way. Without these computations, the results are not correct. In particular, the resulting accumulation and depletion of individual elements strongly influence the local opacities. We have computed the consequences of introducing these effects in stellar models, especially for A-type stars.

\section{Numerical computations}

The stellar models were computed using the Toulouse-Geneva Evolution Code (TGEC), which includes atomic diffusion with radiative accelerations calculated for 21 species, namely 12 elements and their main isotopes: $\mathrm{H},{ }^{3} \mathrm{He},{ }^{4} \mathrm{He},{ }^{6} \mathrm{Li},{ }^{7} \mathrm{Li},{ }^{9} \mathrm{Be},{ }^{10} \mathrm{~B},{ }^{12} \mathrm{C},{ }^{13} \mathrm{C},{ }^{14} \mathrm{~N},{ }^{15} \mathrm{~N},{ }^{16} \mathrm{O},{ }^{17} \mathrm{O},{ }^{18} \mathrm{O},{ }^{20} \mathrm{Ne}$, ${ }^{22} \mathrm{Ne},{ }^{24} \mathrm{Mg},{ }^{25} \mathrm{Mg},{ }^{26} \mathrm{Mg},{ }^{40} \mathrm{Ca}$, and ${ }^{56} \mathrm{Fe}$ ([5]). The diffusion computations were based on the Boltzmann equation for a dilute collision dominated plasma. The details of the physical parameters and computational methods included in the TGEC are described in several papers, including [5]. OPCD v3.3 codes and data ([4]) are used to compute Rosseland opacity at each time step to take the variations of the abundances of each element with depth and time into account. The opacity data are used to compute individual radiative accelerations on $\mathrm{C}, \mathrm{N}, \mathrm{O}, \mathrm{Ne}, \mathrm{Mg}, \mathrm{Ca}$, and $\mathrm{Fe}$. This is done by using the improved semi-analytical prescription proposed by [1] and [3]. The models were evolved from 
pre-main sequence up to hydrogen core exhaustion. Atomic diffusion was introduced at the beginning of the main sequence.

Fingering (thermohaline) convection was also computed. This instability occurs in stars in case of inverse $\mu$-gradients. This may be due to several effects, including accretion of heavy matter onto stars, or accumulation of heavy elements in specific stellar layers due to atomic diffusion. The first treatments of fingering convection in stars were purely analytical. Recently, 2D and 3D numerical simulations were performed (see references in [2]). In the present work, the prescriptions obtained by the most recent 3D simulations ([6]) have been used. They show that the accumulation of heavy elements in specific layers inside A stars leads to important extra mixed zones.

\section{Results}

We present important consequences of atomic diffusion obtained in $1.7 M_{\odot}$ models. Iron accumulates at the place where it is the main contributor of the overall opacity, which creates an important local opacity increase. This opacity increase may lead to a dynamical convective zone induced by iron $\kappa$ mechanism. When fingering convection is taken into account, the dynamical convective zone is less efficient, but in this case new mixed zones due to the fingering (thermohaline) effect do appear. One of them is due to the local calcium accumulation which occurs around $\log (\Delta M / M)=7.5$, as the result of the rapid upward decrease of the radiative acceleration on this element. We computed the surface abundances obtained in these models, to compare them with those observed in several Am stars. When the specific fingering mixed zones are taken into account, the abundances remain somewhat different from the observed ones. However, if we assume that these mixed zones are connected, we find abundances very close to the observed ones (see [2] for more detailed results)

\section{Conclusion}

Atomic diffusion, which is generally considered as a second order process inside stars, may have important macroscopic consequences on the stellar structure, on the hydrodynamic instabilities as well as on the surface abundances. It leads to a local increase of the opacity which must be taken into account when computing the stellar internal structure, the triggering of stellar oscillations and the oscillation frequencies. This needs stellar evolution codes including these effects, which are not trivial. Fingering convection, the only macroscopic process directly induced by the local accumulation of heavy elements in specific stellar layers, has to be taken into account in the evolution of A-type stars. It is an unavoidable consequence of the diffusion-induced inverse $\mu$-gradient. We show that, if this process is the only hydrodynamic instability taken into account in the computations, the stellar gas below the outer convective zones is locally mixed in several layers, which move with time during stellar evolution. If we assume that these zones are dynamically connected, we find a very good fit between the computed and observed abundances.

\section{References}

[1] Alecian, G., \& Leblanc, F., IAU Symp., 224, 587 (2004)

[2] Deal, M., Richard, O., \& Vauclair, S., A\&A, 589, 140 (2016)

[3] Leblanc, F., \& Alecian, G., MNRAS, 352, 1329 (2004)

[4] Seaton, M. J., MNRAS, 362, L1 (2005)

[5] Théado, S., Alecian, G., LeBlanc, F., \& Vauclair, S., A\&A, 546, 100 (2012)

[6] Zemskova, B., Garaud, P., Deal, M., \& Vauclair, S., ApJ, 795, 118 (2014) 